Article

\title{
An Efficiency-Optimized Isolated Bidirectional DC-DC Converter with Extended Power Range for Energy Storage Systems in Microgrids
}

\section{Xiaolong Shi *, Jiuchun Jiang and Xintao Guo}

School of Electrical Engineering, Beijing Jiaotong University, Beijing 100044, China; E-Mails: jcjiang@bjtu.edu.cn (J.J.); 11121655@bjtu.edu.cn (X.G.)

* Author to whom correspondence should be addressed; E-Mail: xiaolong3166@gmail.com; Tel.: +86-138-111-81598; Fax: +86-10-5163-8907.

Received: 19 October 2012; in revised form: 29 November 2012 / Accepted: 14 December 2012 / Published: 24 December 2012

\begin{abstract}
This paper proposes a novel extended-single-phase shift (ESPS) control strategy of isolated bidirectional full-bridge DC-DC converters (IBDCs) which are a promising alternative as a power electronic interface in microgrids with an additional function of galvanic isolation. Based on the mathematical models of ESPS control under steady-state conditions, detailed theoretical and experimental analyses of IBDC under ESPS control are presented. Compared with conventional single-phase-shift (CSPS) control, ESPS control can greatly improve the efficiency of IBDCs in microgrids through decreasing current stress and backflow power considerably over a wide input and output voltage range under light and medium loads. In addition, ESPS control only needs to adjust one single phase-shift angel to control transmission power, thus it retains implementation simplicity in comparison with dual-phase-shift (DPS) control for microgrid applications. Furthermore, an efficiency-optimized modulation scheme based on ESPS and CSPS control is developed in the whole power range of IBDC for power distribution in microgrids. A $10 \mathrm{~kW}$ IBDC prototype is constructed and the experimental results validate the effectiveness of the proposed control strategy, showing that the proposed strategy can enhance the overall efficiency up to $30 \%$.
\end{abstract}

Keywords: isolated bidirectional DC-DC converter; microgrid; energy storage systems; transmission power; backflow power; single-phase-shift control; current stress 


\section{Introduction}

Microgrids have raised great interest in recent years due to the rapid depletion of fossil fuels, rising demand for electricity power, and government policies. Many types of renewable and non-renewable energy sources, such as photovoltaic (PV), wind energy and fuel cell stacks have been widely utilized as distributed energy resources in microgrids [1,2]. However, electric power generated by renewable energy sources is unstable in nature, so the the incorporation of renewable energy is limited in many ways by the variable and intermittent nature of its output. Hence energy storage systems (ESS), such as lithium-ion batteries, super capacitors and electric vehicles which can be distributed energy storage units in intelligent grid [3], have to be used to smooth the source variations [4].

In microgrids, interconnected loads, distributed energy resources, and distributed energy storage systems can be well conjugated and integrated to realize power distribution between energy generation systems and storage systems by bidirectional DC-DC converters (BDCs). Also, galvanic isolation for $\mathrm{BDC}$ is required for the flexibility of system reconfiguration, meeting safety standards, voltage matching and galvanic isolation between the utility grid and the energy storage systems [2,5-8]. Therefore, bidirectional DC-DC converters with galvanic isolation have been proposed as the interface between high-voltage busses with distributed energy resources and low-voltage busses with energy storage devices in microgrids, etc., as shown in Figure 1 [9].

Figure 1. Typical application of IBDC for power distribution in a microgrid.

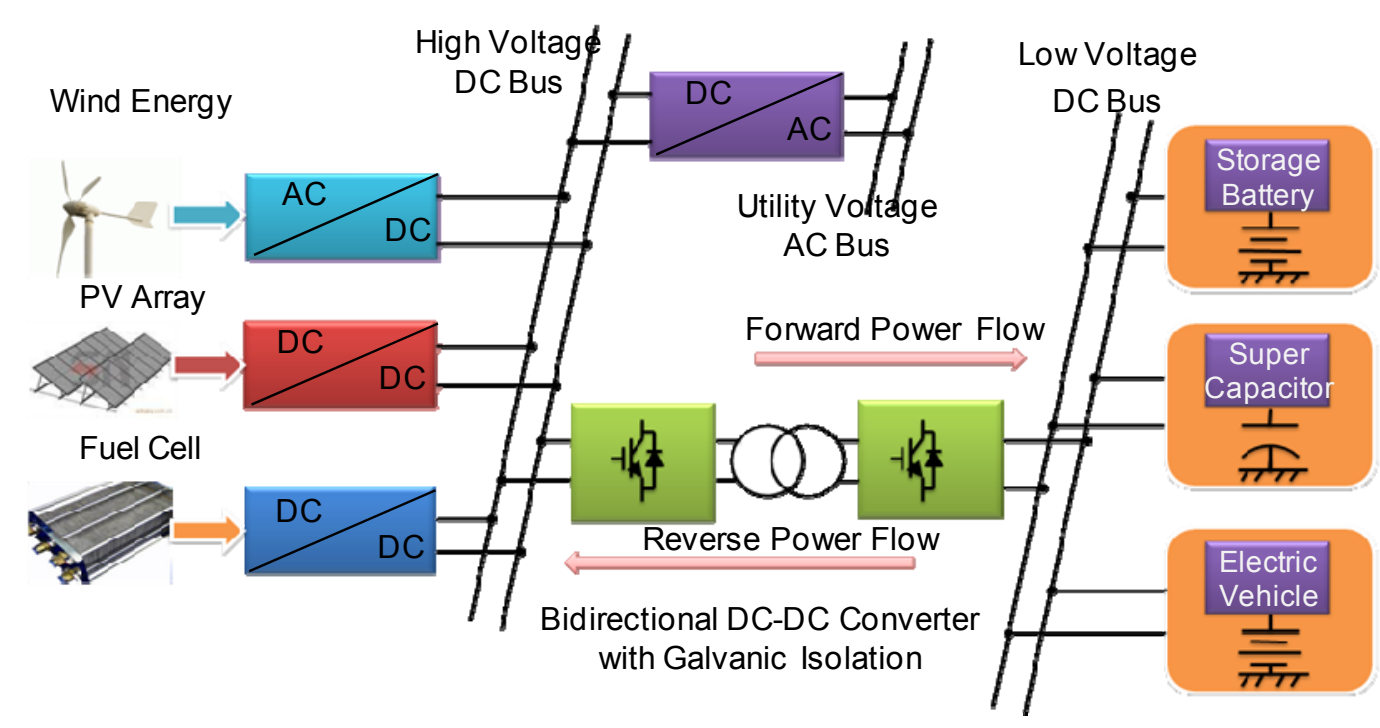

Existing energy storage system employs a $50 \mathrm{~Hz}$ or $60 \mathrm{~Hz}$ transformer for voltage matching and galvanic isolation [2], replacing the line-frequency transformer with a high-frequency isolated bidirectional DC-DC converter (IBDC) would make the energy storage systems more compact and flexible. Due to its soft-switching properties, high power density, high reliability and low number of passive components [10-13], the IBDC based on the single-phase and H-bridge topology with a high-frequency isolation transformer has become a promising alternative as a power electronic interface in microgrids [1,2], as shown in Figure 2. In [4] the authors proposed a parallel topology for IBDCs in microgrids to provide many advantages such as reduced current ripple, higher reliability, and increased capacity. 
IBDC is generally needed to actively control the power flow between energy storage and the load while regulating bus voltage as energy source and load voltage changes. Since energy source and load voltage are not always regulated, it further requires the IBDC to have the capability to deliver power over a wide input voltage and output power range. For instance, the operating voltage range of the Maxwell $48 \mathrm{~V}$ series super capacitor is between $28 \mathrm{~V}$ to $45 \mathrm{~V}$. The load power demand varies between $\mathrm{P}_{\min }=200 \mathrm{~W}$ and $\mathrm{P}_{\max }=1200 \mathrm{~W}$ [14]. Besides, energy systems of electrical vehicles (EV) and storage batteries in microgrids normally consist of numerous typical low-voltage-type batteries in series or parallel strings with wide voltage and power ranges. Therefore, an efficiency-optimized isolated bidirectional DC-DC converter with high voltage diversity is also a key component for charging and discharging the batteries.

Figure 2. Energy storage system based on IBDC in a microgrid.

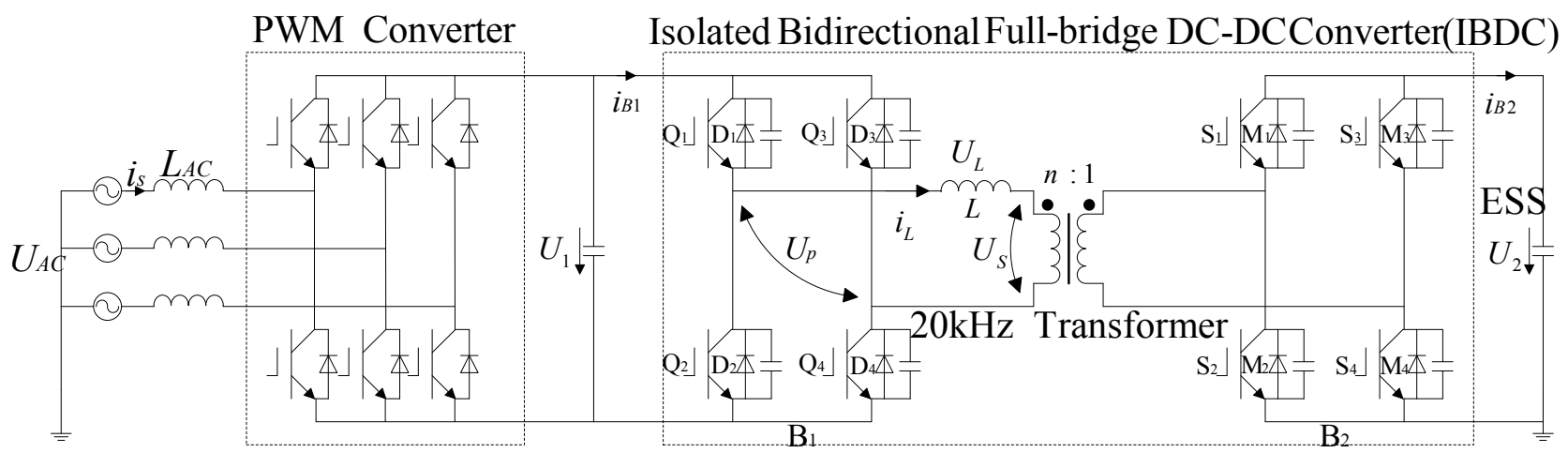

Generally, the most common control methods for such a topology are conventional single-phase-shift (CSPS) control and dual-phase-shift (DPS) control [13-22]. In CSPS control, the gate signals of the corresponding switches in the primary and the secondary bridges are phase-shifted [5,13]. Though this control strategy is simple and convenient, it has only one single variable which can only manage the average output power. As a result, the control flexibility is limited. Other variables, such as backflow power [15], reactive power, and current stress, are unable to be considered in adjusting the phase-shift angle. Therefore, IBDC has extremely low efficiency in working with a wide range of source voltage levels and light load power levels, and it is the primary drawback for the applications in microgrids, as mentioned above [15-17]. The authors in [10,15] and [16] proposed and analyzed a dual-phase-shift strategy (DPS) which includes the phase-shift between the primary and secondary sides as well as duty ratio in each side. The DPS has two degrees of freedom to control the transmission power, which can improve the performance of the IBDC with high voltage diversity under light load compared with CSPS control. However, this proposal needs large amounts of complex expressions to obtain phase-shift pairs at different power ranges [23-24], which is too complex for closed-loop feedback control design and implement on an IBDC in a microgrid. If we take the deadband effects into consideration, the complexity of DPS control would have risen still further.

This study developed the extended-single-phase-shift (ESPS) control of IBDC for power distribution in microgrid to enhance the system efficiency, which is a very intuitive way to realize closed-loop control and easy to implement in engineering. It is mainly implemented in a large voltage 
diversity range to eliminate the backflow power and current stress significantly, thus it reduces the system losses of the IBDC and boosts the overall efficiency. The hybrid modulation scheme is also proposed based on ESPS and CSPS control for the whole power range. Besides, it can also be extended easily to other power conversion systems with a wide range of input and output voltages.

\section{Phase Shift Operation in ESPS Control}

The main waveforms of IBDC in CSPS control are shown in Figure 3, where $T$ is a half switching period, $D_{1}$ represents the phase-shift ratio between the primary and secondary voltages of transformer in CSPS control, where $0 \leq D_{1} \leq 1$.

Figure 3. Waveforms of IBDC in CSPS control.

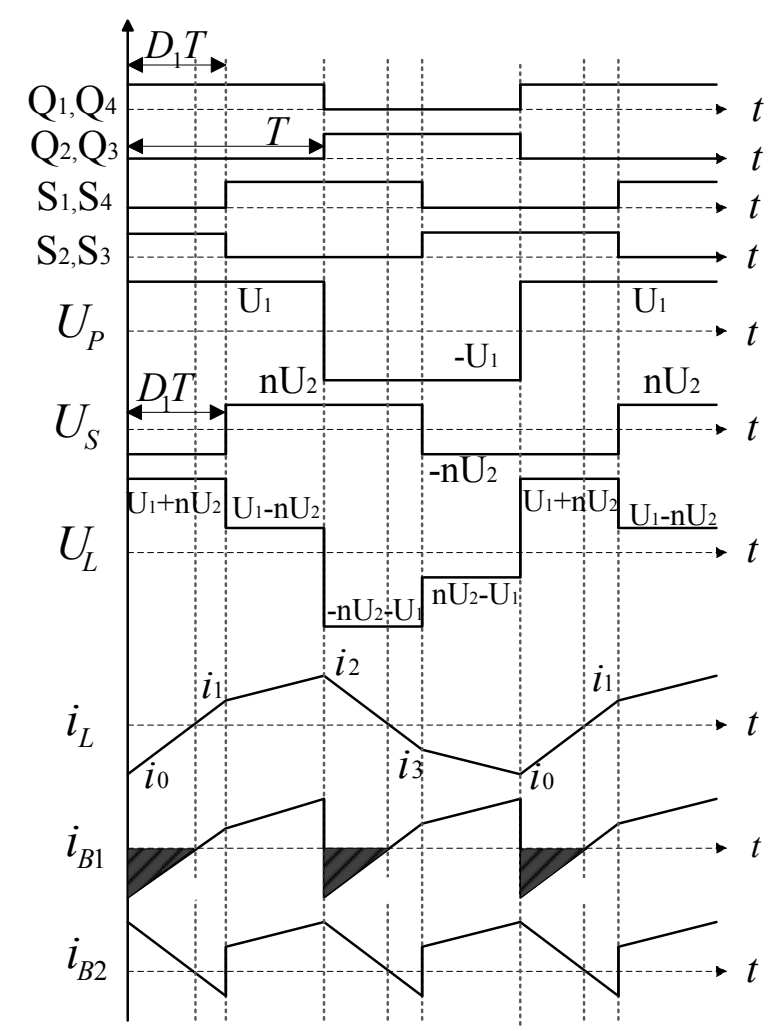

The ESPS control regulates power flow through adjusting the time sequence between the driving signals of diagonal semiconductor switches in the bridge which has larger DC voltage, e.g., $\left(\mathrm{Q}_{1}, \mathrm{Q}_{4}\right)$ in Figure 2, while the driving signals of diagonal semiconductor switches in the bridge with lower DC voltage e.g., $\left(S_{1}, S_{4}\right)$ are synchronous, and they are also the same as one of the driving signals of corresponding semiconductor switches e.g., $\left(\mathrm{Q}_{1}\right.$ or $\left.\mathrm{Q}_{4}\right)$ in the other bridge.

Figure 4(a) shows the ideal waveforms of the ESPS control in buck operation when the power flows from $U_{1}$ to $U_{2}$ according to the variables shown in Figure 2, and we assume $U_{1} \geq n U_{2}$ in Figure 4(a). In Figure $4(\mathrm{a})$, the $50 \%$ duty-cycle gate driving signals of $\mathrm{Q}_{1}$ is shifted ahead of $\mathrm{Q}_{4}$ by the phase-shift angle $\alpha$ which is positive. The driving signals of $S_{1}, S_{4}$ and $\mathrm{Q}_{4}$ are synchronous, and the driving signals of $\mathrm{Q}_{2}, \mathrm{Q}_{3}, S_{2}, S_{3}$ are complementary $50 \%$ duty cycle signals. In the reverse power flow, the driving signal of $\mathrm{Q}_{1}$ is just need to shift to the opposite direction. As shown in Figure 4(a), $U_{P}$ is a three-level voltage, we define $D$ as high-level ratio of the three-level pulse width modulation (PWM) voltage 
waveform, where $0 \leq D \leq 1, \alpha=\pi-D \pi, U_{S}$ is a $50 \%$ duty-cycle square wave, which has the same rising edge as $U_{P}$. In contrast, Figure 4(b) shows the ideal waveforms of the ESPS control in boost mode when the power flows from $U_{1}$ to $U_{2}$. The conducting switches for buck and boost modes are also shown in Figure 4.

Overall, ESPS control only need to adjust a single variable, which is simple and easy to realize in closed-loop control like CSPS control, also it is convenient to implement on IBDC in microgrids. For the sake of convenience, the power flow direction is assumed from $U_{1}$ to $U_{2}$ in this paper.

Figure 4. Waveforms of IBDC in ESPS control. (a) Buck mode; (b) Boost mode.

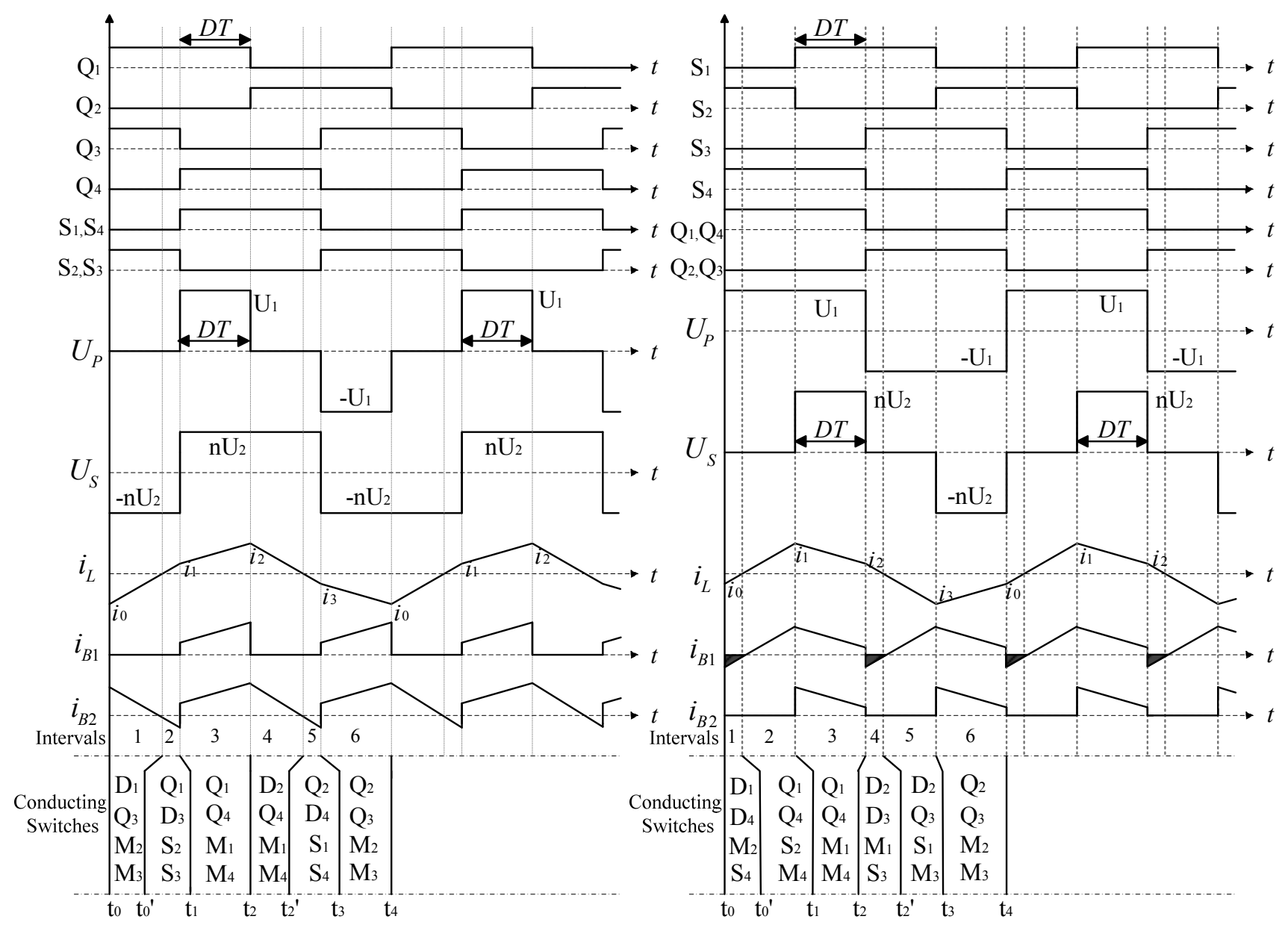

(a)

(b)

\section{Mathematical Models of ESPS Control in Steady-State Conditions}

The average transmission power of IBDC with ESPS control can be obtained by solving the following expression:

$$
P=\frac{1}{T} \int_{0}^{T} U_{P}(t)^{*} I_{L}(t) d t
$$

where $T=1 / 2 f, f$ is the switching frequency. The transmission power can be calculated as:

$$
P=\frac{n U_{2} U_{1}}{4 f L} D(1-D)
$$


where $n$ is the turn ratio of the transformer, $L$ is the leakage inductance, $U_{1}$ and $U_{2}$ are input voltage and output voltage, respectively, and $D$ is the high-level ratio. The output current can be expressed as:

$$
I=\frac{n U_{1}}{4 f L} D(1-D)
$$

When the load is set as resistance $R$, from (2), the output voltage becomes:

$$
U_{2}=\frac{n U_{1} R}{4 f L} D(1-D)
$$

Equations (2), (3) and (4) are valid for both buck and boost modes, the constraint is $0 \leq D \leq 1$. The instantaneous current of different intervals for buck and boost mode can be obtained by solving (5) and (6), respectively, according to current and voltage waveforms of leakage inductance. The instantaneous currents of typical moments in Figure 4 and the peak current are shown in Table 1 and Table 2, respectively:

$$
\begin{aligned}
& i_{0}+\frac{n U_{2}}{2 f L}(1-D)+\frac{U_{1}-n U_{2}}{2 f L} D=-i_{0} \\
& i_{0}+\frac{U_{1}}{2 f L}(1-D)+\frac{U_{1}-n U_{2}}{2 f L} D=-i_{0}
\end{aligned}
$$

Table 1. The instantaneous currents of leakage inductance with ESPS control.

\begin{tabular}{cccc}
\hline $\boldsymbol{i}_{\boldsymbol{L}}(\boldsymbol{t})$ & $\boldsymbol{t}=\boldsymbol{t}_{0}$ & $\boldsymbol{t}=\boldsymbol{t}_{1}$ & $\boldsymbol{t}=\boldsymbol{t}_{2}$ \\
\hline Buck & $\frac{1}{4 f L}\left(2 n D U_{2}-U_{1} D-n U_{2}\right)$ & $\frac{1}{4 f L}\left(n U_{2}-D U_{1}\right)$ & $\frac{1}{4 f L}\left(U_{1} D+n U_{2}-2 n D U_{2}\right)$ \\
Boost & $\frac{1}{4 f L}\left(n D U_{2}-U_{1}\right)$ & $\frac{1}{4 f L}\left((1-2 D) U_{1}+n D U_{2}\right)$ & $\frac{1}{4 f L}\left(U_{1}-n D U_{2}\right)$ \\
\hline
\end{tabular}

Table 2. The peak currents of leakage inductance with ESPS control.

\begin{tabular}{ccc}
\hline Condition & $\boldsymbol{U}_{1} \geq \boldsymbol{n} \boldsymbol{U}_{2}$ & $\boldsymbol{U}_{1}<\boldsymbol{n} \boldsymbol{U}_{2}$ \\
\hline Buck & $i_{\max }=\frac{D U_{1}+(1-2 D) n U_{2}}{4 f L}$ & $i_{\max }=\frac{-D U_{1}+n U_{2}}{4 f L}$ \\
Boost & $i_{\max }=\frac{U_{1}-n D U_{2}}{4 f L}$ & $i_{\max }=\frac{(1-2 D) U_{1}+n D U_{2}}{4 f L}$ \\
\hline
\end{tabular}

The rms current of buck and boost operation can be derived by solving (7) and (8), respectively, as shown in (9):

$$
\begin{gathered}
I_{\mathrm{rms}-1}=\sqrt{\frac{1}{T}\left[\int_{0}^{(1-D) T}\left(i_{0}+\frac{n U_{2}}{L} t\right)^{2} d t+\int_{(1-D) T}^{T}\left(i_{1}+\frac{U_{1}-n U_{2}}{L}(t-T+D T)\right)^{2} d t\right]} \\
I_{\mathrm{rms}-2}=\sqrt{\frac{1}{T} \int_{0}^{T} i_{L}^{2} d t}=\sqrt{\frac{1}{T}\left[\int_{0}^{(1-D) T}\left(i_{0}+\frac{U_{1}}{L} t\right)^{2} d t+\int_{(1-D) T}^{T}\left(i_{1}+\frac{U_{1}-n U_{2}}{L}(t-(1-D) T) d t\right]\right.}
\end{gathered}
$$




$$
I_{\mathrm{rms}}= \begin{cases}\frac{1}{12 f L} \sqrt{12 n D^{3} U_{1} U_{2}-6 D^{3} U_{1}^{2}-18 n D^{2} U_{1} U_{2}+9 D^{2} U_{1}^{2}+3 n^{2} U_{2}^{2}} & \text { buck } \\ \frac{1}{12 f L} \sqrt{12 n D^{3} U_{1} U_{2}-6 n^{2} D^{3} U_{2}^{2}-18 n D^{2} U_{1} U_{2}+9 n^{2} D^{2} U_{2}^{2}+3 U_{1}^{2}} \text { boost }\end{cases}
$$

Similarly, from Figure 3, the transmission power of IBDC under CSPS control is:

$$
P^{\prime}=\frac{n U_{2} U_{1}}{2 f L} D_{1}\left(1-D_{1}\right), 0<D_{1}<1
$$

The output current is:

$$
I_{o}^{\prime}=\frac{n U_{1}}{2 f L} D_{1}\left(1-D_{1}\right), 0<D_{1}<1
$$

The rms current, peak current with CSPS control are derived as (12):

$$
\begin{aligned}
& I_{r m s}^{\prime}=\frac{1}{12 f L} \sqrt{36 n D_{1}^{2} U_{1} U_{2}-24 n D_{1}^{3} U_{1} U_{2}+3 U_{1}^{2}-6 n U_{1} U_{2}+3 n^{2} U_{2}^{2}} \\
& I_{\text {max }}^{\prime}= \begin{cases}\frac{1}{4 f L}\left(-n U_{2}+U_{1}+2 n U_{2} D_{1}\right) & \text { buck } \\
\frac{1}{4 f L}\left(n U_{2}-U_{1}+2 U_{1} D_{1}\right) & \text { boost }\end{cases}
\end{aligned}
$$

\section{Analysis and Comparisons of ESPS and CSPS Control}

\subsection{Comparative Analysis of Transmission Power}

For the convenience of analysis, all quantities in this section are normalized by following base values:

$$
U_{b}=U_{1} \quad I_{b}=\frac{U_{1}}{\omega L} \quad P_{b}=\frac{U_{1}^{2}}{\omega L}
$$

The normalized output power and current referred to the primary side are:

$$
\begin{aligned}
& P[p u]=\left\{\begin{array}{l}
\frac{\pi d}{2} D(1-D), 0<D<1, \text { ESPS } \\
\pi d D_{1}\left(1-D_{1}\right), 0<D_{1}<1, \text { CSPS }
\end{array}\right. \\
& i_{o}[p u]=\left\{\begin{array}{l}
\frac{\pi}{2} D(1-D), 0<D<1, \text { ESPS } \\
\pi D_{1}\left(1-D_{1}\right), 0<D_{1}<1, \text { CSPS }
\end{array}\right.
\end{aligned}
$$

where $d$ is the voltage conversion ratio of the IBDC, $d$ [pu] $=d=n U_{2} / U_{1} \cdot g$ is system parameter, $g=n^{2} R / \omega L$, which includes all the design parameters, it can also be derived by $g=d / I_{O}[\mathrm{pu}]$. 
Figure 5. Comparative analysis of transmission power. (a) 3-D curve of normalized transmission power; (b) 2-D curve of normalized output current.

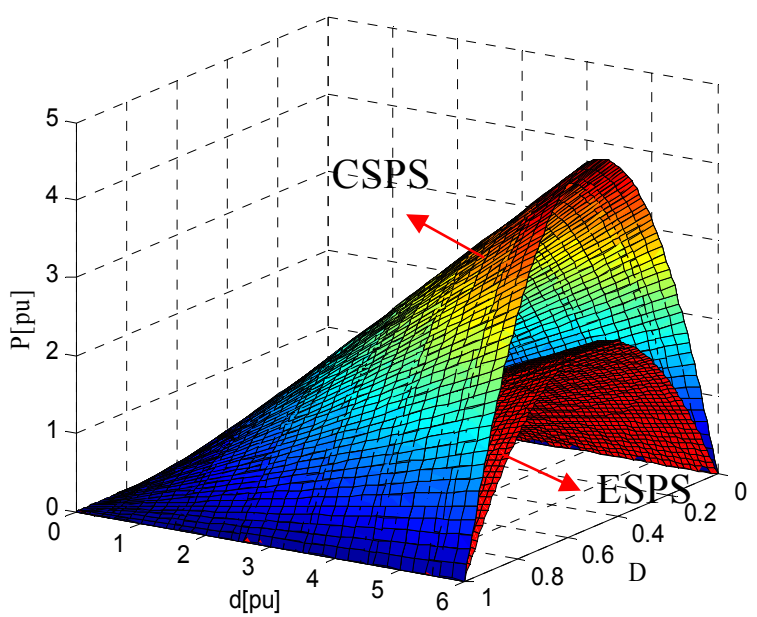

(a)

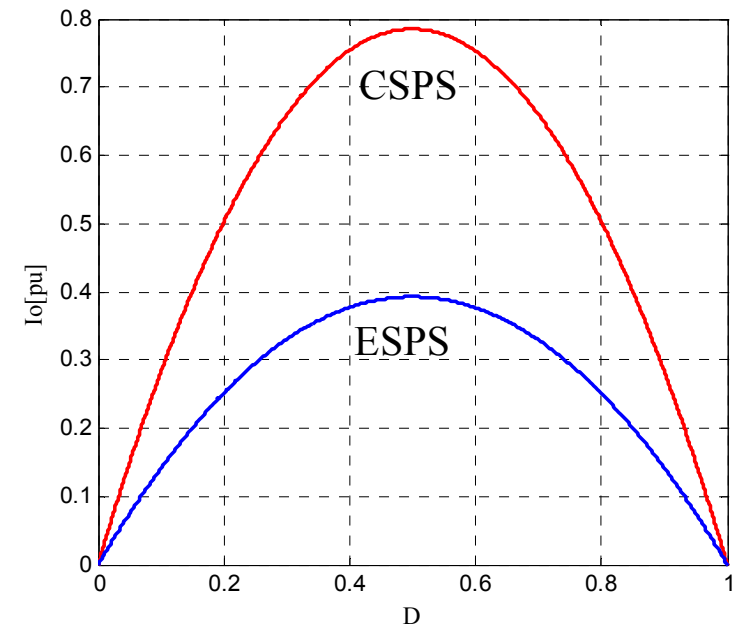

(b)

Figure 5 shows the comparative analysis of transmission power between ESPS and CSPS control. The output power and current varies according to high-voltage ratio $D$ in ESPS control, and the maximum normalized output current is $0.393 \mathrm{pu}$, the maximum transmission power can be achieved when $D=0.5$, which is similar with CSPS control, as the output current range in the ESPS control is half of that in CSPS control, thus ESPS control are mainly implemented under light and medium load to overcome the inherent disadvantages of IBDC in CSPC control. When the output power is beyond the power range of ESPS control, it can be switched to CSPS control easily to realize full range operation instead of DPS control for the reason that the DPS control achieves limited improvement of efficiency compared with CSPS control for higher load conditions [8,16,18,25].

\subsection{The Algorithm to Implement ESPS in Light and Medium Load with Wide Voltage Ratio Range}

The IBDC in CSPS control has the inherent disadvantage of high current stress and reactive power, especially in extremely low and high operating voltage ranges with low output current, resulting in great system losses and extremely low system efficiency. While the voltage ratio $d$ is close to 1 , the current stress and reactive power in CSPS control is almost the lowest compared with other operating ranges. Therefore, a good way to design the converter is to select a value of $n$ to have $d=1$ under nominal conditions where the IBDC can achieve the highest efficiency. However, in microgrids, the IBDC is required to have the capability to deliver power over wide input voltage and output power ranges, and the IBDC should keep a high system efficiency when the value of $d$ deviates far from 1, especially under light and medium loads. Thus ESPS control is proposed to solve the problem properly.

Figure 6 shows the nomogram to implement ESPS and the hybrid modulation scheme for IBDC with extended power range in microgrids for energy storage applications, curves of different constant value of $g$, which include all the design parameters, are also added. ESPS control is implemented mainly in low and high voltage level regions for light and medium load conditions, which will be analyzed in the next section. As the maximum transmission power in ESPS control is half of that in 
CSPS control, when the transmission power is beyond the range of ESPS control, the modulation strategy should be switched to CSPS control as shown in Figure 6, since CSPS control is supposed to be the best operation mode for high-power operation $[8,10,16,18,25]$.

Figure 6. Hybrid modulation scheme for IBDC with extended power range.

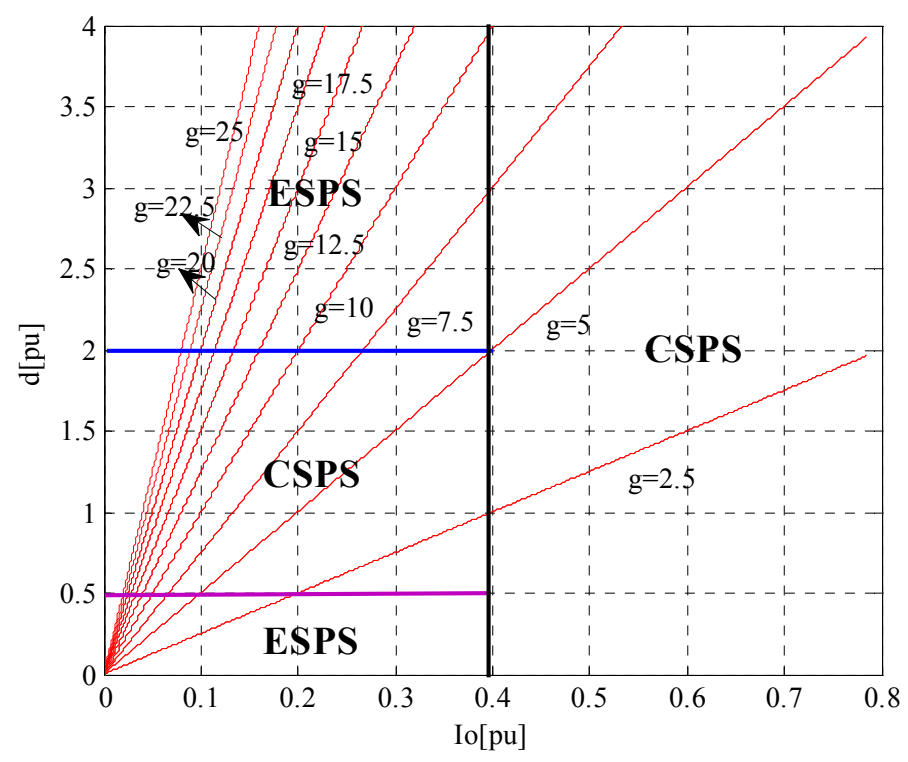

\subsection{Comparative Analysis of Current Stress}

The basic prerequisite for comparative analysis of current stress is that the transmission power of ESPS and CSPS control are the same. Figure 7 shows curves of the normalized peak current varied with $I_{o}$ and $d$. The duty-ratio of CSPS control is set to $0 \leq D_{1} \leq 0.5$ and the high voltage ratio of ESPS control in this paper is also set to $0 \leq D \leq 0.5$.

Figure 7. Curves of the normalized peak current varied with $I_{o}$ and $d$. (a) 3-D curve, $0 \leq d \leq 0.5$; (b) 3 -D curve, $d \geq 2$; (c) 2-D curve, $0 \leq d \leq 0.5$; (d) 2-D curve, $d \geq 2$.

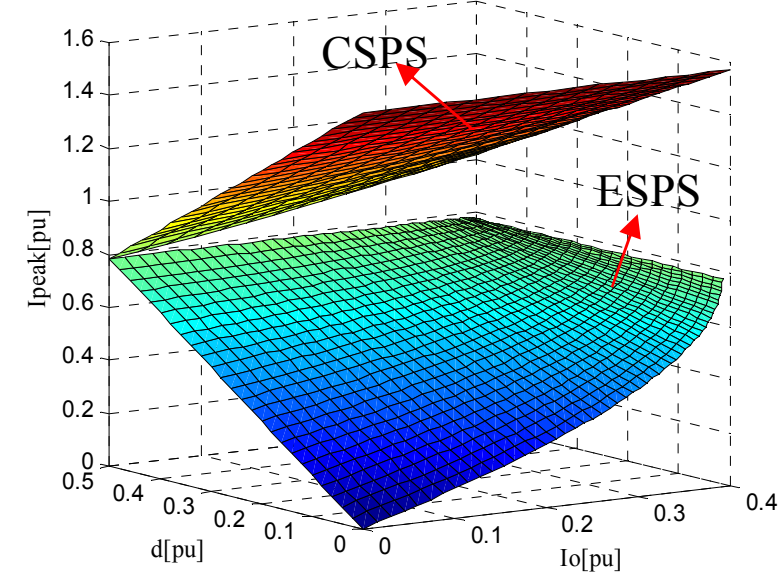

(a)

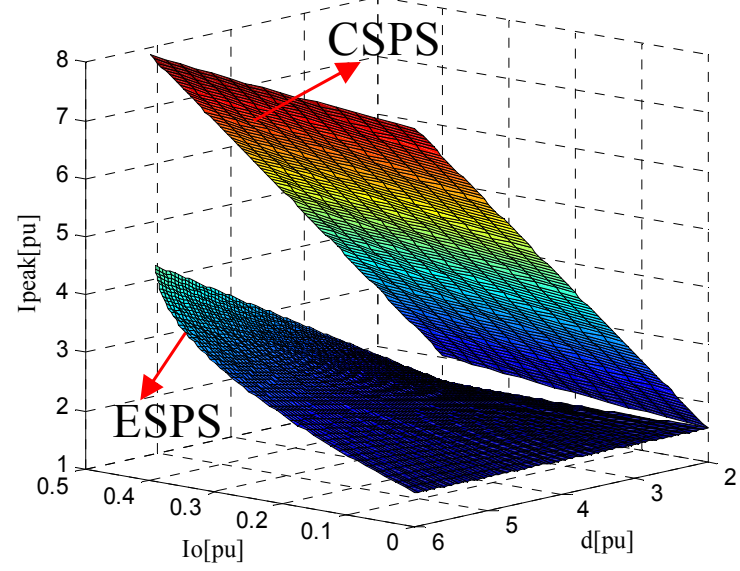

(b) 
Figure 7. Cont.

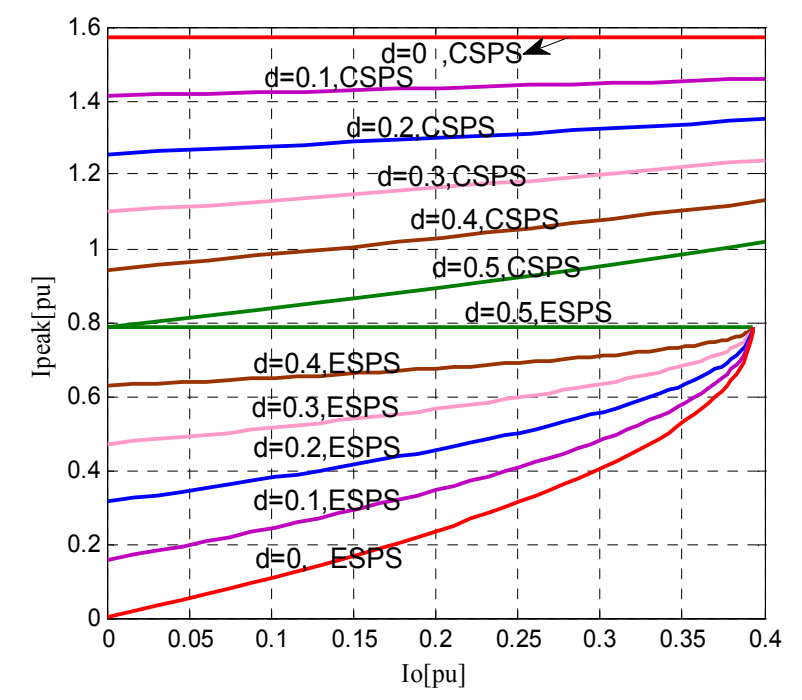

(c)

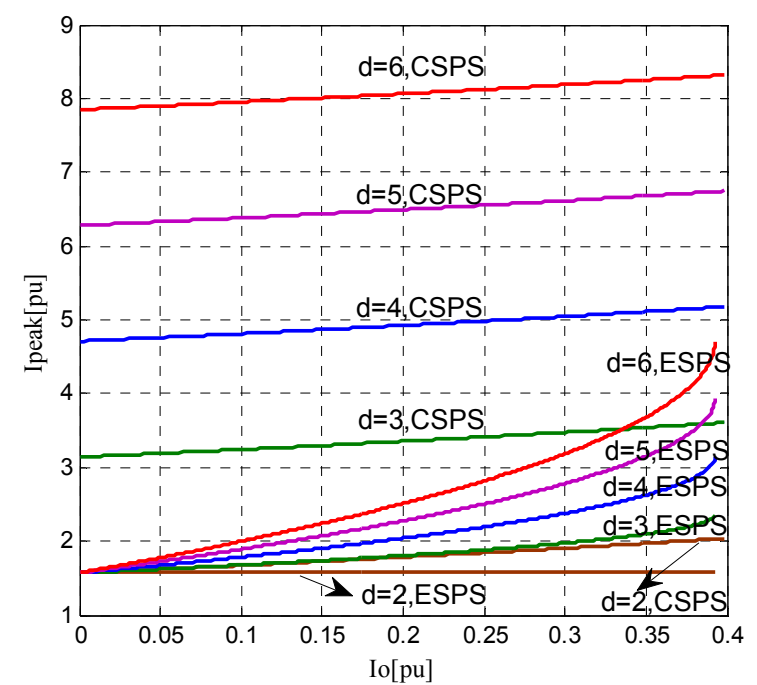

(d)

As shown in Figures $7(\mathrm{a}, \mathrm{c})$, for the same output voltage and current, when $0 \leq d \leq 0.5, i_{r m s}$ in ESPS control significantly decreased in the whole power range, especially in much lower voltage ratio range. In Figures 7(b,d), ESPS control is also advantageous compared with CSPS control when $d \geq 2$, and $i_{r m s}$ is much lower than CSPS while voltage ratio $d$ increases. Thus IBDC with ESPS control has far lower current stress in the whole defined operation regions in Figure 6.

High current stress results in an unnecessary electrical stress on the semiconductor switches and transformer of the IBDC, especially under light-load operation and wide voltage ratio conditions with CSPS control [15]. Besides, the primary energy losses of IBDCs includes switching losses and conduction losses. The copper loss of the transformer and the conduction loss of switching devices are both proportional to the rms current of leakage inductance, and larger peak current results in larger core losses and switching losses [25]. Thus it is of great significance to reduce current stress to optimizethe system efficiency with ESPS control in microgrids where wide variations in input and output are expected.

\subsection{The Comparative Analysis of Backflow Power}

The leakage inductance is an instantaneous energy storage element of an IBDC converter. However, the phase of the primary current is not always the same as the primary voltage, as shown in Figure 3. Thus the portion that is sent back to the primary voltage source $U_{1}$ is defined as IBDC backflow power [15], namely the dark-shaded area in Figure 3 and Figure 4. The backflow power can be derived by the following expression:

$$
P_{b}=\frac{1}{T} U_{1} \int_{t_{0}}^{t_{0}^{\prime}} i(t) d t
$$

According to Table 1 , when $0 \leq d \leq 0.5, i_{0}$ is always negative, while $i_{1}>0$, namely $D<d$, the backflow power is zero, while $i_{1} \leq 0$, namely $D \geq d$, the backflow power can be derived as (17). 
Similarly, when $d>2$, the backflow power can be derived as (18). As for CSPS control, the backflow power is derived as (19) according to current directions of $i_{0}$ and $i_{1}$ in Figure 3 :

$$
\begin{gathered}
P_{f}= \begin{cases}\frac{U_{1}\left(n U_{2}-U_{1} D\right)^{2}}{16 f L\left(U_{1}-n U_{2}\right)} & i_{0}<0, i_{1} \leq 0 \\
0 & i_{0}<0, i_{1}>0\end{cases} \\
P_{f}= \begin{cases}\frac{\left(U_{1}-n U_{2} D\right)^{2}}{16 f L} & i_{1}>0, i_{0} \leq 0 \\
\frac{U_{1}\left(U_{1}-n U_{2} D\right)^{2}}{16 f L\left(n U_{2}-U_{1}\right)} & i_{1}>0, i_{0}>0\end{cases} \\
P_{f}= \begin{cases}\frac{U_{1}\left(n U_{2}-U_{1}+2 n U_{2} D_{1}\right)^{2}}{16 f L\left(U_{1}-n U_{2}\right)}+\frac{U_{1}\left(U_{1}-n U_{2}\right)\left(1-D_{1}\right) D_{1}}{16 f L} & i_{0}<0, i_{1} \leq 0 \\
\frac{U_{1}\left(n U_{2}-U_{1}-2 n U_{2} D_{1}\right)^{2}}{16 f L\left(U_{1}+n U_{2}\right)} & i_{0} \leq 0, i_{1}>0 \\
\frac{U_{1}\left(n U_{2}-U_{1}-2 n U_{2} D_{1}\right)^{2}}{16 f L\left(n U_{2}-U_{1}\right)} & i_{0}>0, i_{1}>0\end{cases}
\end{gathered}
$$

The backflow power derived above can be normalized by (13). Figure 8 shows the comparison of backflow power between ESPS and CSPS control according to different voltage ratio ranges. When $0 \leq d \leq 0.5$, the backflow power in ESPS control is extremely low in the whole defined power range, and the backflow power is zero in some regions, as shown in Figure 8(a) and (c). When $d \geq 2$ the backflow power is also declined significantly with ESPS control, for instance, when $d=5$, $i_{o}=0.15 \mathrm{pu}$, the normalized backflow power of ESPS control is only $0.1 \mathrm{pu}$ while it rises to $1.2 \mathrm{pu}$ in CSPS control as shown in Figure 8(b) and (d). Overall, ESPS control can decrease the backflow power dramatically in the whole power range when $0 \leq d \leq 0.5$ and $d \geq 2$.

Figure 8. Curves of the normalized backflow power varied with $I_{o}$ and $d$. (a) 3-D curve, $0 \leq d \leq 0.5$; (b) 3-D curve, $d \geq 2$. (c) 2-D curve, $0 \leq d \leq 0.5$; (d) 2-D curve, $d \geq 2$.

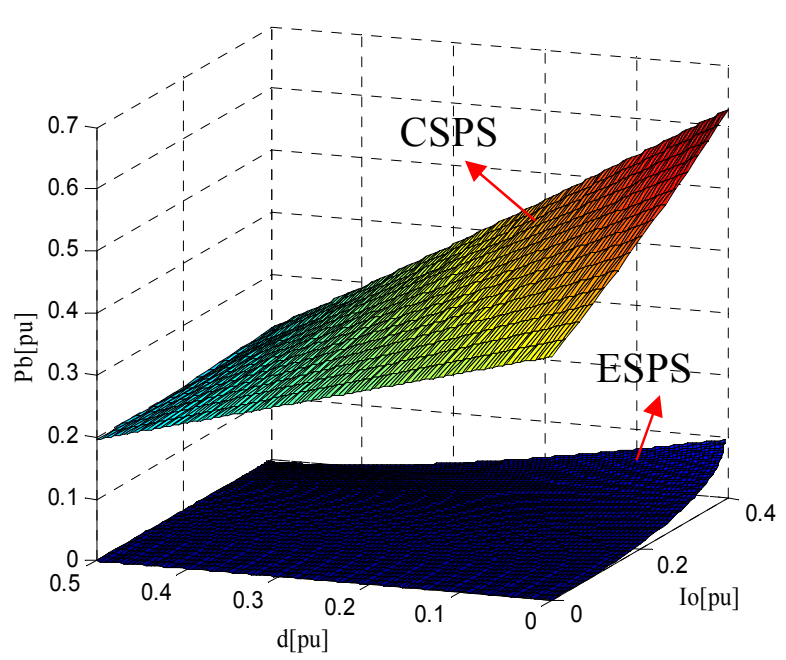

(a)

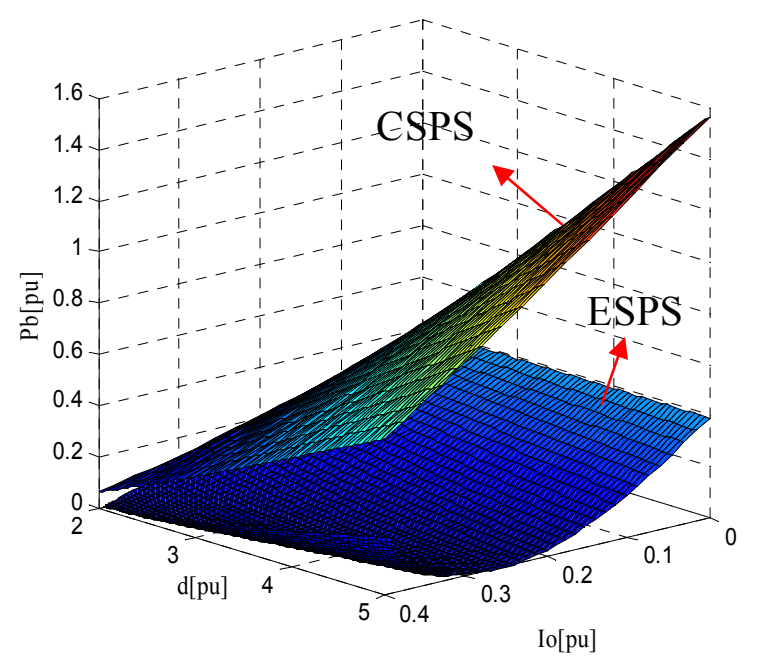

(b) 
Figure 8. Cont.

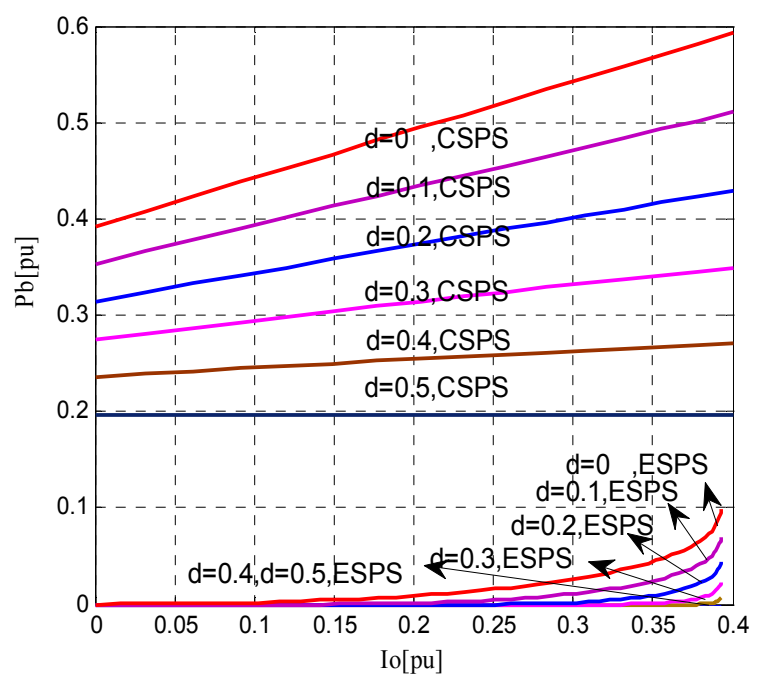

(c)

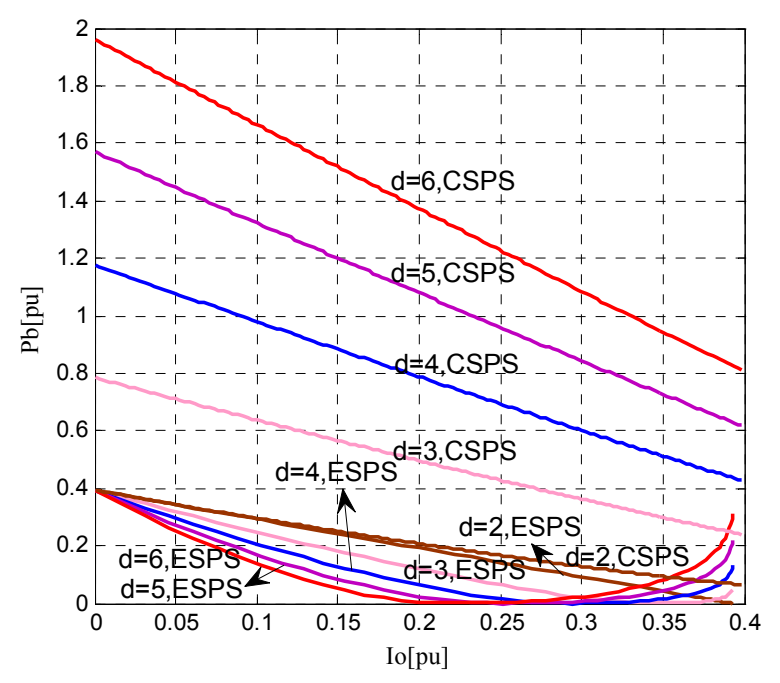

(d)

For a given transmission power, with the increase of the backflow power, the forward power also increases to compensate the losses caused by backflow power. Then the circulating power and current stress increase, resulting in great losses in power devices and magnetic components as well as low efficiency of the IBDC in microgrids, especially for low-power output operations [15]. Thus it is also of great significance to decrease the backflow power through implementing ESPS control to enhance the overall efficiency of IBDC in microgrids.

\section{Experimental Results}

In order to verify the aforementioned analysis, a $10 \mathrm{~kW}$ prototype of IBDC topology is constructed. The main parameters of converter are shown in Table 3.

Table 3. The parameters of prototype.

\begin{tabular}{cc}
\hline Output Power $\left(\boldsymbol{P}_{\boldsymbol{O}}\right)$ & $\mathbf{0}-\mathbf{1 0 ~ k W}$ \\
\hline Switching Frequency $(f)$ & $20 \mathrm{kHz}$ \\
Input DC Voltage $\left(U_{1}\right)$ & $100-500 \mathrm{~V}$ \\
Output DC Voltage $\left(U_{2}\right)$ & $0-500 \mathrm{~V}$ \\
Transformer Turns Ratio $(n)$ & $1: 1$ \\
Series Inductance $(L)$ & $120 \mu \mathrm{H}$ \\
Output Load $(R)$ & $25 / 200 \Omega$ \\
\hline
\end{tabular}

Figure 9 shows a photograph of the actual prototype and configuration of the experimental set. The IBDC is made of H-bridge modules BSM150GB120DN2, IGBT module from Infineon. The digital controller to run the proposed modulation strategy is implemented using DSP TMS320F28335 from Texas Instruments. 
Figure 9. Photograph of the IBDC converter prototype and configuration of the converter.

(a) The IBDC converter prototype; (b) Configuration of the experimental set.

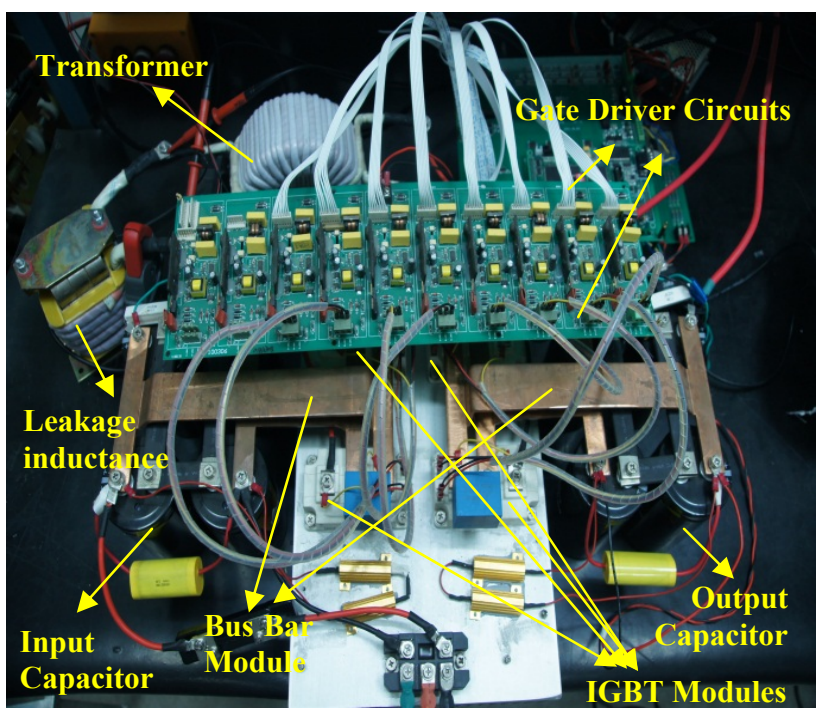

(a)

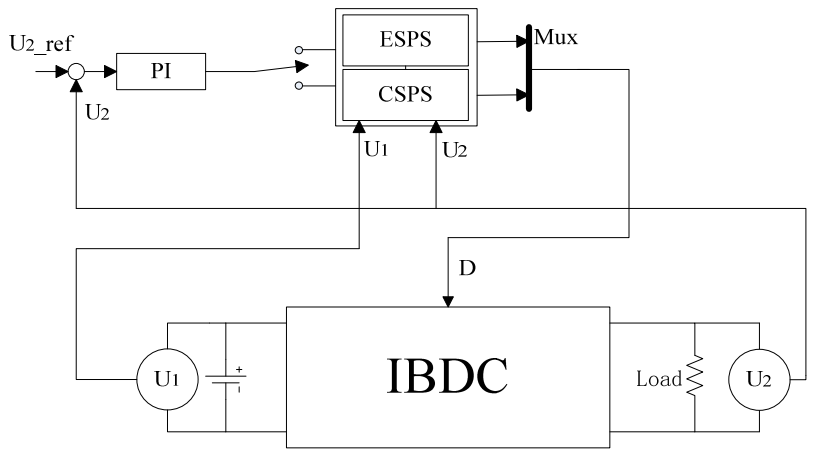

(b)

\subsection{The Comparisons of ESPS and CSPS Control in Low Voltage Ratio Range}

In order to verify performance analysis of both ESPS and CSPS control in the low voltage ratio range, the input voltage and output load are specified as $500 \mathrm{~V}$ and $25 \Omega$, respectively, the output voltage varies from 0 to $250 \mathrm{~V}$, namely the voltage ratio $d$ is set as $d \leq 0.5$. Figure 10 shows the peak current and backflow power of IBDC varied with transmission power in both modulation strategies as well as the theoretically calculated results based on the mathematical models built in Section 3.

Figure 10. Curves of the current stress and backflow power in both modulation strategies varied with $d$. (a) Peak current; (b) Backflow power.

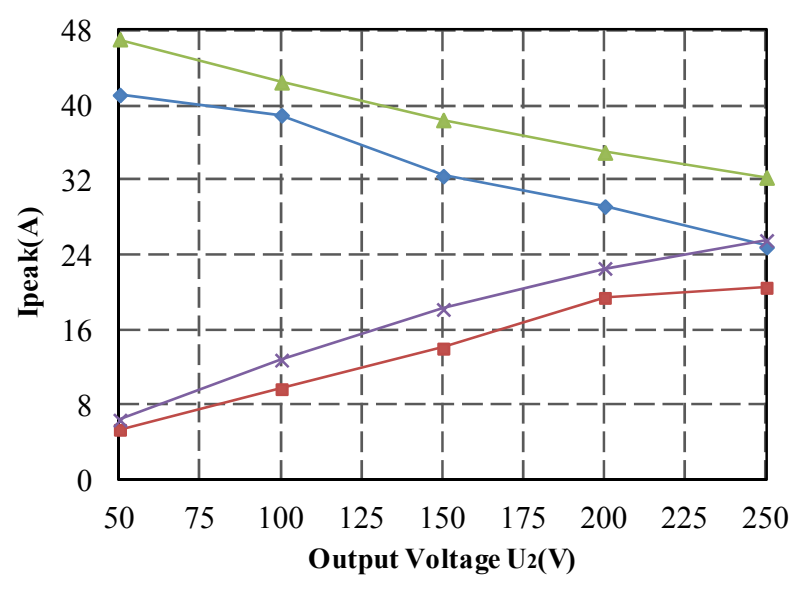

$\neg$ Measured,CSPS $\rightarrow-$ Measured,ESPS

(a)

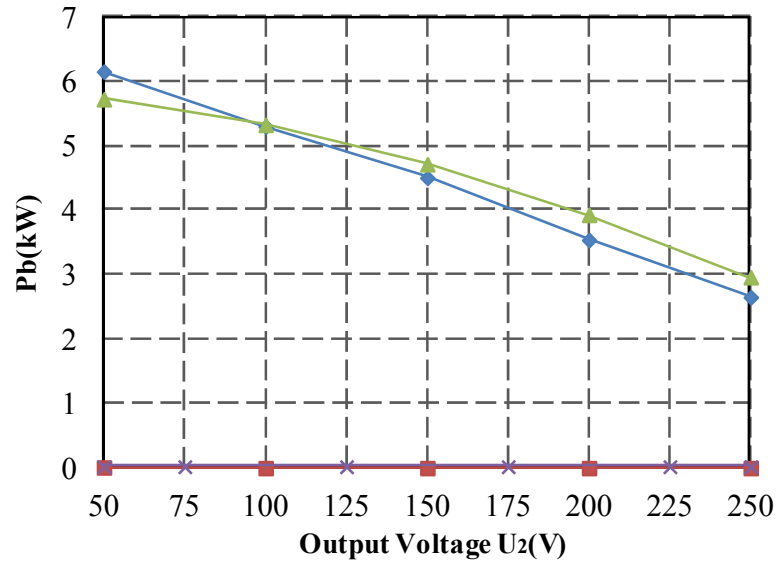

- Calculated,CSPS $\quad *$ Calculated,ESPS

(b) 
The experimental waveforms of ESPS and CSPS control for the same transmission power are shown in Figure 11.

Figure 11. The experimental waveforms of ESPS and CSPS control when output voltages $U_{2}$ are $50 \mathrm{~V}$ and $100 \mathrm{~V}$. (a) ESPS control with $U_{1}=500 \mathrm{~V}, U_{2}=50 \mathrm{~V}$; (b) CSPS control with $U_{1}=500 \mathrm{~V}, U_{2}=50 \mathrm{~V}$; (c) ESPS control with $U_{1}=500 \mathrm{~V}, U_{2}=100 \mathrm{~V}$; (d) CSPS control with $U_{1}=500 \mathrm{~V}, U_{2}=100 \mathrm{~V}$.

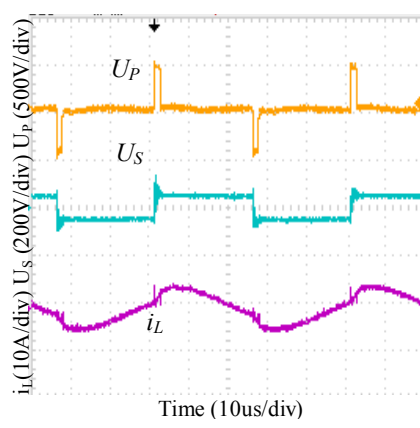

(a)

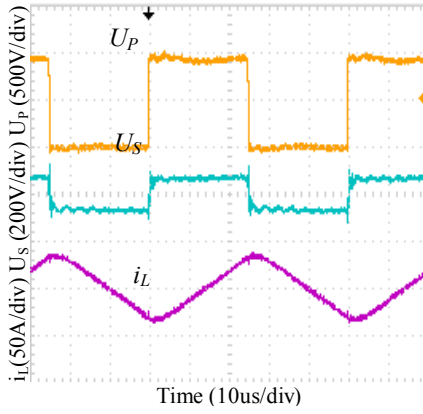

(b)

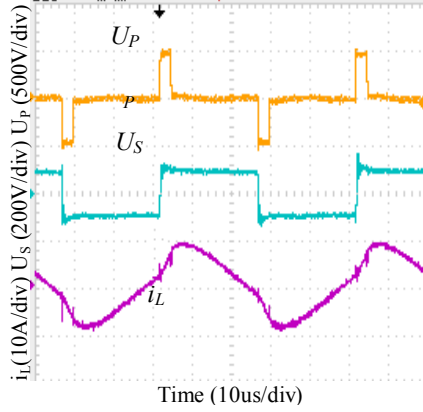

(c)

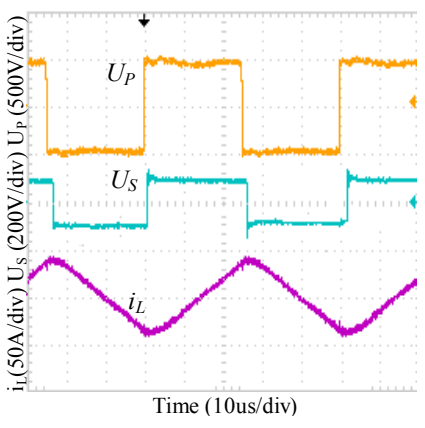

(d)

As can be seen in Figure 10, the measured results of both ESPS and CSPS control present a good agreement with the theoretically calculated results, which verifies the mathematical models very well. It can be observed in Figure 10(a) that the current stress of components is significantly reduced with ESPS control in comparison with CSPS control, especially when the voltage ratio $d$ deviates from 0.5. Figure 10(b) shows that the backflow power keeps zero with ESPS control while it is as high as $6 \mathrm{~kW}$ with CSPS control for the same transmission power. Thus it can be concluded that the efficiency of IBDC can be significantly improved by reducing system losses in low voltage ratio range. Figure 12 shows the efficiency curves of IBDC in both control methods. It can be easily found that the ESPS control can achieve much higher efficiency than the CSPS control for the same transmission power, and the improvement can be up to $30 \%$ in low voltage ratio range.

Figure 12. Comparison of system efficiency between CSPS and ESPS control.

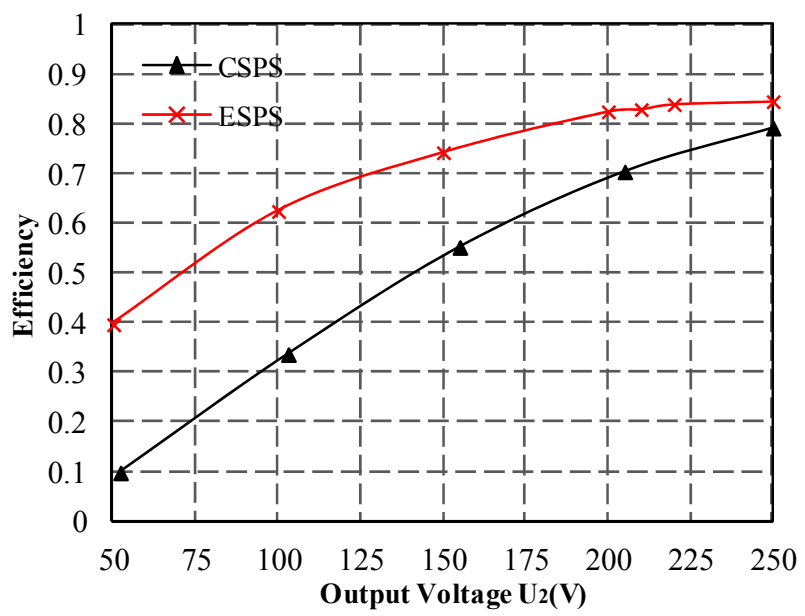




\subsection{The Comparisons of ESPS and CSPS Control in High Voltage Ratio Range}

In order to verify the performance of ESPS in the high voltage ratio range, under the same experiment prototype, the input voltage is set for $100 \mathrm{~V}$ while the output voltage range is varied from 100 to $500 \mathrm{~V}$, namely the voltage ratio range is $1 \leq d \leq 5$. The performance comparison between ESPS and CSPS control in the high voltage ratio range (for $d \geq 2$ ) is shown in Figure 13, the theoretically calculated results of both control methods are also presented for verification. As can be seen from Figure 13(a) and Figure 13(b), the current stress and backflow power with ESPS control are decreased dramatically compared with CSPS control, especially when the voltage conversion ratio is much larger than 2, Figure 14 shows the voltage and current waveforms of leakage inductance with ESPS and CSPS control, which also well validates the theoretical analysis.

Figure 13. Curves of the current stress and reactive power varied with $d$ in ESPS and CSPS control. (a) Peak current; (b) Backflow power.

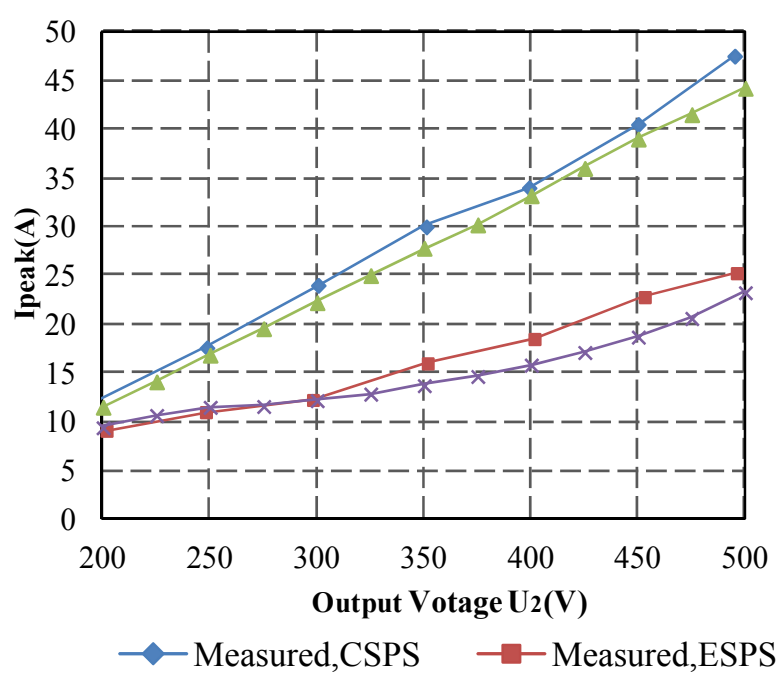

(a)

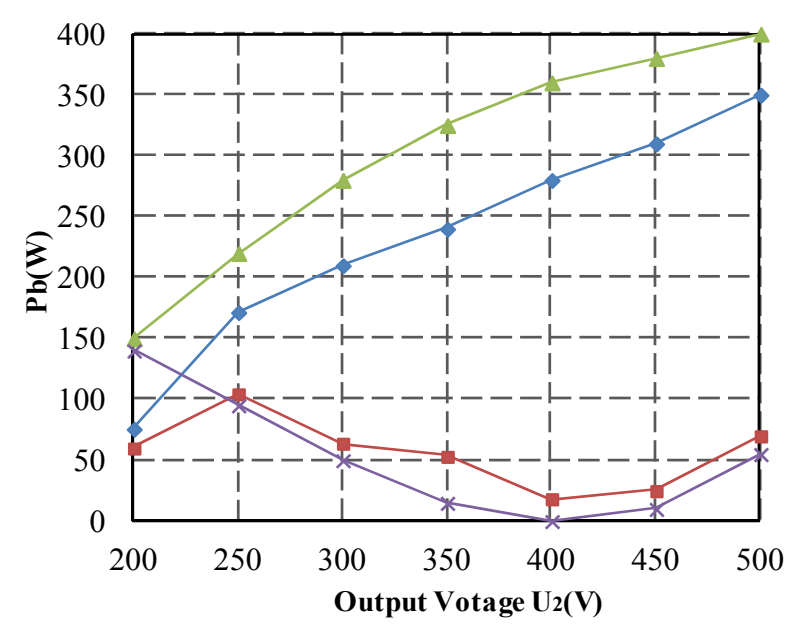

$\times$ Calculated,ESPS

(b)

Figure 14. The experimental waveforms of ESPS and CSPS control when output voltages $U_{2}$ are $250 \mathrm{~V}$ and $500 \mathrm{~V}$. (a) ESPS control with $U_{1}=100 \mathrm{~V}, U_{2}=250 \mathrm{~V}$; (b) CSPS control with $U_{1}=100 \mathrm{~V}, U_{2}=250 \mathrm{~V}$; (c) ESPS control with $U_{1}=100 \mathrm{~V}, U_{2}=500 \mathrm{~V}$; (d) CSPS control with $U_{1}=100 \mathrm{~V}, U_{2}=500 \mathrm{~V}$.

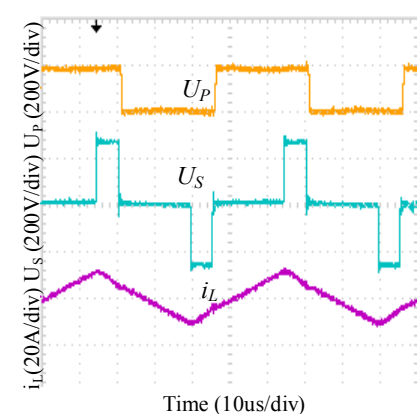

(a)

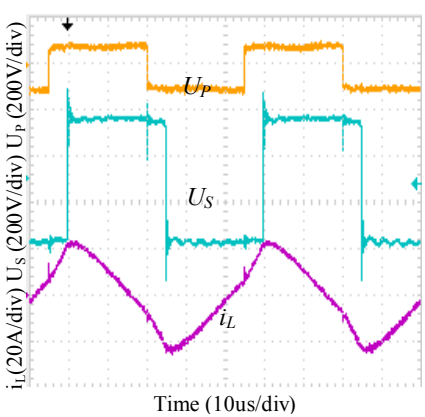

(b)

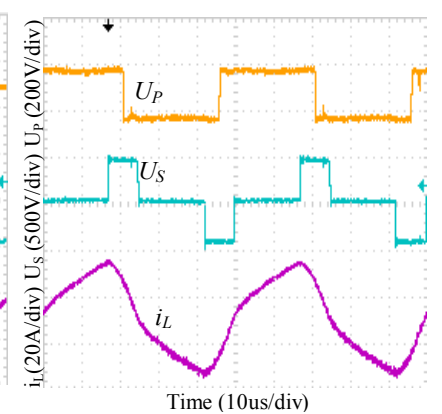

(c)

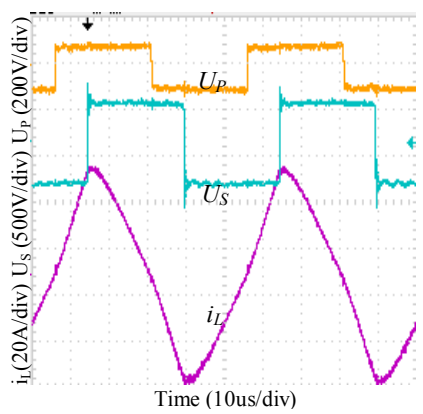

(d) 
The overall efficiency of IBDC is the highest when the voltage ratio $d$ is nearly 1 [13-17], and it drops dramatically when $d$ deviates from 1 with CSPS control. Figure 15 shows the system efficiency with both controls when $2 \leq d \leq 5$ as well as the efficiency of IBDC in CSPS control when $1 \leq d \leq 2$. As can be seen in Figure 15, the system efficiency is high for the nominal output voltage when the voltage ratio $d$ is 1 with CSPS control, however it decreases significantly in the large voltage ratio range. In contrast, the overall efficiency of IBDC with ESPS control remains more than $20 \%$ higher than that of CSPS control in the large voltage ratio range, and the improvement can be up to $30 \%$ when the voltage ratio $d$ is 3 , which also verifies the aforementioned analysis pretty well.

Figure 15. Comparison of system efficiency with CSPS and ESPS control when $2 \leq d \leq 5$.

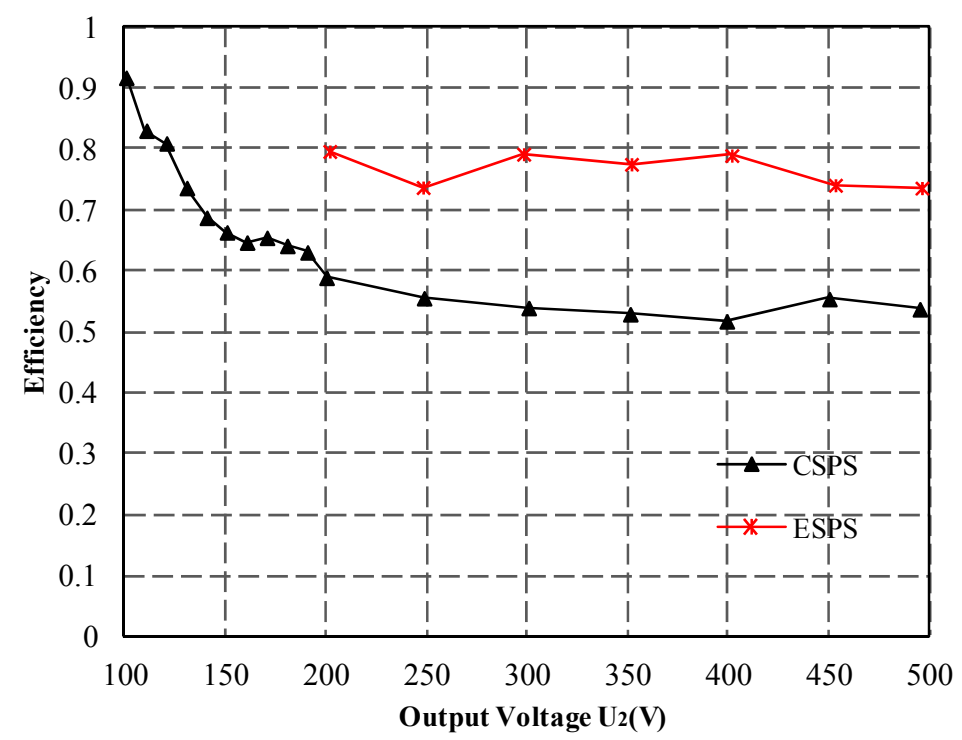

\section{Conclusions}

This paper proposes the ESPS control of IBDC in order to improve the system efficiency of IBDC for power distribution between energy source and energy storage systems in microgrids over a wide input voltage and output power range. The current stress and backflow power of IBDC under ESPS and CSPS control are comparatively analyzed to verify the superiority of ESPS control, showing that the advantages are particularly striking for microgrid applications with wide variation in input or output voltages.

A series of experiments test based on a $10 \mathrm{~kW}$ laboratory prototype were carried out to demonstrate the practical performance of the theoretical proposal. From the theoretical analysis and the experimental results, it can be concluded that ESPS control has the following features:

1. ESPS control reduces both current stress and backflow power dramatically when $d<0.5$ and $d>2$ under light and medium load, thus saving costs and reducing system losses considerably.

2. The overall system efficiency is significantly optimized in the defined operation region.

3. ESPS control only needs to control one single variation, thus it is very simple in principle and easy to implement for microgrid applications.

4. The hybrid modulation scheme based on ESPS and CSPS control is an advanced control strategy to ensure high system efficiency of IBDC with extended power range in microgrids while retaining implementation simplicity. 


\section{Acknowledgments}

The work was supported by the National Natural Science Foundation of China (No. 51277010) and National High Technology Research and Development Program of China (No. 2012AA050211).

\section{References}

1. Inoue, S.; Akagi, H. A bidirectional isolated DC-DC converter as a core circuit of the next-generation medium-voltage power conversion system. IEEE Trans. Power Electron. 2007, 22, 535-542.

2. Inoue, S.; Akagi, H. A bidirectional DC-DC converter for an energy storage system with galvanic isolation. IEEE Trans. Power Electron. 2007, 22, 2299-2306.

3. Wang, X.J.; Tian, W.Q.; He, H.J.; Huang, M.; Jiang, J.C.; Han, H.Y. The application of electric vehicles as mobile distributed energy storage units in smart grid. In Proceedings of Asia-Pacific Power Energy Engineering Conference, Wuhan, China, 25-28 March 2011.

4. Li, W.; Joos, G.; Abbey, C.A. A parallel bidirectional DC/DC converter topology for energy storage systems in wind applications. In Proceedings of IEEE Industry Applications Society Annual Meeting (IAS), New Orleans, LA, USA, 23-27 September 2007; pp. 179-185.

5. De Doncker, R.W.; Divan, D.M.; Kheraluwala, M.H. A three-phase soft-switched high power-density Dc/Dc converter for high-power applications. IEEE Trans. Ind. Appl. 1991, 27, 63-73.

6. Kim, J.; Song, H.S.; Nam, K. Asymmetric duty control of a dual-half-bridge DC/DC converter for single-phase distributed generators. IEEE Trans. Power Electron. 2011, 26, 973-982.

7. Xie, Y.; Sun, J.; Freudenberg, J.S. Power flow characterization of a bidirectional galvanically isolated high-power DC/DC converter over a wide operating range. IEEE Trans. Power Electron. 2010, 25, 54-66.

8. Naayagi, R.T.; Forsyth, A.J.; Shuttleworth, R. High-Power bidirectional DC-DC converter for aerospace applications. IEEE Trans. Power Electron. 2012, 27, 4366-4379.

9. Chen, W.; Rong, P.; Lu, Z.Y. Snubberless bidirectional DC-DC converter with new CLLC resonant tank featuring minimized switching loss. IEEE Trans. Ind. Electron. 2010, 57, 3075-3086.

10. Zhao, B.; Song, Q.; Liu, W.H. Power characterization of isolated bidirectional dual-active-bridge DC-DC converter with dual-phase-shift control. IEEE Trans. Power Electron. 2012, 27, 4172-4176.

11. Wu, K.Y.; . De. Silva, C.W.; Dunford, W.G. Stability analysis of isolated bidirectional dual active full-bridge DC-DC Converter with triple phase-shift control. IEEE Trans. Power Electron. 2012, 27, 2007-2017.

12. Wai, R.J.; Duan, R.Y. High-efficiency bidirectional converter for power sources with great voltage diversity. IEEE Trans. Power Electron. 2007, 22, 1986-1996.

13. Kheraluwala, M.H.; Gascoigne, R.W.; Divan, D.M.; Baumann, E.D. Performance characterization of a high-power dual active bridge Dc-to-Dc converter. IEEE Trans. Ind. Appl. 1992, 28, 1294-1301.

14. Zhou, H.; Khambadkone, A.M. Hybrid modulation for dual active bridge bi-directional converter with extended power range for ultracapacitor application. IEEE Trans. Ind. Appl. 2009, 45, 1434-1442. 
15. Bai, H.; Mi, C. Eliminate reactive power and increase system efficiency of isolated bidirectional dual-active-bridge DC-DC converter using novel dual-phase-shift control. IEEE Trans. Power Electron. 2008, 23, 2905-2914.

16. Oggier, G.G.; Garcia, G.O.; Oliva, A.R. Switching control strategy to minimize dual active bridge converter losses. IEEE Trans. Power Electron. 2009, 24, 1826-1838.

17. Zhang, J.; Lai, J.S.; Kim, R.Y.; Yu, W. High-power density design of a soft-switching high-power bidirectional Dc-Dc converter. IEEE Trans. Power Electron. 2007, 22, 1145-1153.

18. Segaran, D.; Holmes, D.G.; McGrath, B.P. Comparative analysis of single and three-phase dual active bridge bidirectional DC-DC converters. In Proceedings of Australian Universities Power Engineering Conference, Sydney, Australia, 14-17 December 2008; pp. 1-6.

19. Bai, H.; Nie, Z.; Mi, C. Experimental comparision of traditional phase-shift, dual-phase-shift, and model-based control of isolated bidirectional dc-dc converters. IEEE Trans. Power Electron. 2010, $25,1444-1449$.

20. Krismer, F.; Kolar, J.W. Closed form solution for minimum conduction loss modulation of IBDC converters. IEEE Trans. Power Electron. 2012, 27, 174-188.

21. Oggier, G.G.; Garcia, G.O.; Oliva, A.R. Modulation strategy to operate the dual active bridge DC-DC converter under soft switching in the whole operating range. IEEE Trans. Power Electron. 2011, 26,1228-1236.

22. Guo, X.T.; Jiang, J.C.; Niu, L.Y.; Li, J.X.; Qu, Y.Q. A 10kW DC-DC system based on a bidirectional high-frequency isolated dual active bridge DC-DC topology. In Proceedings of IEEE Power Engineering and Automation Conference, Wuhan, China, 8-9 September 2011; pp. 50-54.

23. Jain, A.K.; Ayyanar, R. PWM control of dual active bridge; Comprehensive analysis and experimental verification. IEEE Trans. Power Electron. 2011, 26, 1215-1227.

24. Krismer, F.; Kolar, J.W. Efficiency-optimized high current dual active bridge converter for automotive applications. IEEE Trans. Ind. Electron. 2012, 59, 2745-2760.

25. Kim, M.; Rosekeit, M.; Sul, S.K.; De Doncker, R.W.A.A. A dual-phase-shift control strategy for dual-active-bridge DC-DC converter in wide voltage range. In Proceedings of 8th International Conference Power Electronics-ECCE Asia, 30 May-3 June 2011; pp. 364-371.

(C) 2013 by the authors; licensee MDPI, Basel, Switzerland. This article is an open access article distributed under the terms and conditions of the Creative Commons Attribution license (http://creativecommons.org/licenses/by/3.0/). 